\title{
PRESERVING MEASURABILITY WITH COHEN ITERATIONS
}

\author{
RADEK HONZÍK \\ Department of Logic, Faculty of Arts, Charles University \\ E-mail: radek.honzik@ff.cuni.cz
}

\begin{abstract}
We describe a weak version of Laver indestructibility for a $\mu$-tall cardinal $\kappa, \mu>\kappa^{+}$, where "weaker" means that the indestructibility refers only to the Cohen forcing at $\kappa$ of a certain length. A special case of this construction is: if $\mu$ is equal to $\kappa^{+n}$ for some $1<n<\omega$, then one can get a model $V^{*}$ where $\kappa$ is measurable, and its measurability is indestructible by $\operatorname{Add}(\kappa, \alpha)$ for any $0 \leq \alpha \leq \kappa^{+n}$ (Theorem 3.3).
\end{abstract}

Keywords: Cohen forcing, measurability

AMS subject code classification: 03E35, 03E55

\section{Introduction}

Assume $\kappa$ is supercompact. In [7], Laver defined an iteration $P$ of length $\kappa$ such that in $V[P],{ }^{1} \kappa$ is still supercompact and every further $\kappa$-directed closed forcing preserves the supercompactness of $\kappa$ ( $P$ is often called the Laver preparation). We also say that $\kappa$ is Laver-indestructible in $V[P]$. The proof of this indestructibility result is essentially based on two useful properties of a supercompact cardinal $\kappa$ in $V$ : (i) for every $\mu \geq \kappa$, one can choose an elementary embedding $j: V \rightarrow M$ with critical point $\kappa$ such that $M$ is closed under $\mu$-sequences existing in $V$; this closure is then used to find a master condition in $M$ and proceed with a lifting argument which ensures that supercompactness is preserved, ${ }^{2}$ (ii) there is a single function $f: \kappa \rightarrow V_{\kappa}$ such that for every $x \in V$, one can choose an embedding $j$ in (i) so that $j(f)(\kappa)=x$ (this $f$ is often called the Laver function).

A typical example of a $\kappa$-directed closed forcing is the Cohen forcing at $\kappa$, which we will denote by $\operatorname{Add}(\kappa, \alpha),{ }^{3}$ where $\alpha$ is any ordinal larger than 0 . The fact that over $V[P]$, $\operatorname{Add}(\kappa, \alpha)$ preserves the measurability of $\kappa$ is very useful when one wishes to use some

$1 \quad V[P]$ indicates a $P$-generic extension of $V$ whenever it is not important to distinguish specific $P$-generic filters. For instance the statement " $\varphi$ holds in $V[P]$ " means that $\varphi$ holds in $V[G]$ for every $P$-generic filter $G$.

2 Assume $j: V \rightarrow M$ is an elementary embedding, $P$ is a forcing notion, $G$ is $P$-generic over $V$, and $H$ is $j(P)$-generic over $M$. Then a sufficient condition for $j$ to lift, i.e. a sufficient condition for the existence of $j^{+}: V[G] \rightarrow M[H]$ with $j^{+} \uparrow V=j$, is that we have $j^{\prime \prime} G \subseteq H$. With supercompactness, we can often argue that $j^{\prime \prime} G$ is a condition in $M$ (a master condition), and $H$ can then be built below this master condition. For more details, see [3].

3 Formally speaking, conditions in $\operatorname{Add}(\kappa, \alpha)$ are partial functions of size $<\kappa$ from $\kappa \times \alpha$ to 2 . The ordering is by reverse inclusion. 
large cardinal properties of $\kappa$ in $V[P][\operatorname{Add}(\kappa, \alpha)]$ (see for instance [4] where a model with the tree property at $\kappa^{++}, \kappa$ strong limit singular with cofinality $\omega$, is constructed starting with a supercompact $\kappa$ ).

A natural question is whether a "Laver-like" indestructibility is available also for smaller large cardinals. As it turns out, it is the property (i) above which is more important: it is known that for instance a strong cardinal ${ }^{4} \kappa$ has the analogue of the Laver function, but it is not known whether it can be made indestructible under $\kappa$-directed closed forcings. ${ }^{5}$

In this short paper we use the idea of Woodin (as described in [2]) to argue that it is possible to have a limited indestructibility of a $\mu$-tall cardinal ${ }^{6} \kappa, \kappa^{+}<\mu$ regular, in the sense that we can successively extend $V \subseteq V^{1} \subseteq V^{*}$ so that forcing with $\operatorname{Add}(\kappa, \mu)$ over $V^{*}$ yields the measurability of $\kappa$. See Section 2 .

If $\mu=\kappa^{+n}, 1<n<\omega$, we can say more. If $\kappa$ is $H\left(\kappa^{+n}\right)$-hypermeasurable ${ }^{7}, V^{*}$ has the property that forcing with $\operatorname{Add}(\kappa, \alpha)$ over $V^{*}$ for $0<\alpha \leq \kappa^{+n}$ yields the measurability, in fact hypermeasurability, of $\kappa$ (Theorem 3.1 and Theorem 3.3). Note that in $V^{*}, \kappa$ may actually stop being measurable ${ }^{8}$ depending on the iteration $P_{\kappa}$ which gives $V^{*}=V^{1}\left[P_{\kappa}\right]$; compare the constructions in Theorem 3.1 and 3.3.

Remark 1.1. We assume that the reader is familiar with the lifting arguments. The general reference is [3]; the more specific constructions used in the present paper are also given in [2].

\section{Tall cardinals}

In this section, we assume GCH. Let $\kappa$ be $\mu$-tall cardinal for some regular $\kappa^{+}<\mu$. Let $j: V \rightarrow M$ be a $\mu$-tall embedding with the extender representation:

$$
M=\{j(f)(\alpha) \mid f: \kappa \rightarrow V \& \alpha<\mu\} .
$$

In particular, $M$ is closed under $\kappa$-sequences in $V$ and $\mu<j(\kappa)<\mu^{+}$. Let $U$ be the normal measure derived from $j$, and let $i: V \rightarrow N$ be the ultrapower embedding generated by $U$. Let $k: N \rightarrow M$ be elementary so that $j=k \circ i$. Note that $\kappa$ is the critical point of $j, i$ and $j, i$ have support $\kappa$, i.e. every element of $M$ and $N$ is of the form $j(f)(\alpha)$, or $i(f)(\kappa)$ respectively, for some $f$ with domain $\kappa$. In contrast, the critical point of $k$ is $\left(\kappa^{++}\right)^{N}$ and $k$ has support which we denote $v$, where $\left(\kappa^{++}\right)^{N}<v<i(\kappa)$, i.e. every element of $M$ can be written as $k(f)(\alpha)$ for some $f$ in $N$ with domain $v{ }^{9}$

Let $P$ denote the forcing $\operatorname{Add}(\kappa, \mu)$ in $V, Q=i(P)$, and let $g$ be a $Q$-generic filter over $V$. Then the following hold:

4 A regular cardinal $\kappa$ is strong if for every $\mu \geq \kappa$ there is $j: V \rightarrow M$ with critical point $\kappa$ and $H(\mu) \subseteq M$.

A non-supercompact strong cardinal $\kappa$ can be indestructible under $\kappa$-directed closed forcings by a method of [1], but $\kappa$ needs to be supercompact in the ground model.

There is $j: V \rightarrow M$ with critical point $\kappa$ such that $M$ is closed under $\kappa$-sequences and $j(\kappa)>\mu$.

$7 \kappa$ is $H(\mu)$-hypermeasurable (also $H(\mu)$-strong) if there is an elementary embedding $j: V \rightarrow M$ with critical point $\kappa$ such that $j(\kappa)>\mu, H(\mu) \subseteq M$, and $M$ is closed under $\kappa$-sequences in $V$.

8 If in $V^{*}, \kappa$ is not measurable, and it is measurable again in $V^{*}[\operatorname{Add}(\kappa, \alpha)]$ (for a specific $\alpha$ ), it is more appropriate to call this step a "resurrection" of the measurability of $\kappa$. $v$ needs to have the property that $k(v) \geq \mu$; some such $v$ always exists. 
Theorem 2.1. GCH. Forcing with Q preserves cofinalities and the following hold in $V[g]$ :

(i) $j$ lifts to $j^{1}: V[g] \rightarrow M\left[j^{1}(g)\right]$, where $j^{1}$ restricted to $V$ is the original $j$.

(ii) $i$ lifts to $i^{1}: V[g] \rightarrow N\left[i^{1}(g)\right]$, where $i^{1}$ restricted to $V$ is the original $i . N\left[i^{1}(g)\right]$ is the measure ultrapower obtained from $j^{1}$.

(iii) $k$ lifts to $k^{1}: N\left[i^{1}(g)\right] \rightarrow M\left[j^{1}(g)\right]$, where $k^{1}$ restricted to $N$ is the original $k$.

(iv) $g$ is $Q$-generic over $N\left[i^{1}(g)\right]$.

Proof. We show that $Q$ is $\kappa^{+}$-closed and $\kappa^{++}$-cc in $V$. Closure is obvious by the fact that $N$ is closed under $\kappa$-sequences in $V$. Regarding the chain condition, notice that every element of $Q$ can be identified with the equivalence class of some function $f: \kappa \rightarrow$ $\operatorname{Add}(\kappa, \mu)$. For $f, g: \kappa \rightarrow \operatorname{Add}(\kappa, \mu)$, set $f \leq g$ if for all $i<\kappa, f(i) \leq g(i)$; it suffices to check that the ordering $\leq$ on these $f$ 's is $\kappa^{++}$-cc. Let $A$ be a maximal antichain in this ordering; take an elementary substructure $\bar{M}$ in some large enough $H(\theta)$ of $V$ which contains all relevant data, has size $\kappa^{+}$and is closed under $\kappa$-sequences. Then it is not hard to check that $A \cap \bar{M}$ is maximal in the ordering (and so $A \subseteq \bar{M}$ ), and therefore has size at most $\kappa^{+}$.

(i) and (ii). These follow by $\kappa^{+}$-distributivity of $Q$ in $V$ and the fact that $j, i$ have support $\kappa$ : the pointwise image of $g$ generates a generic for $j(Q)$ and $i(Q)$, respectively.

(iii). $i(Q)$ is $i\left(\kappa^{+}\right)$-closed in $N$, and since $v<i\left(\kappa^{+}\right)$, we use the distributivity of $i(Q)$ and the fact that $k$ has support $v$ to argue that the pointwise image of $i^{1}(g)$ generates a generic filter which is equal to $j^{1}(g)$ by commutativity of $j, i, k$.

(iv). $Q$ is $i\left(\kappa^{+}\right)$-cc in $N$ and $i(Q)$ is $i\left(\kappa^{+}\right)$-closed in $N$. There are therefore mutually generic over $N$ by Easton's lemma.

Remark 2.2. It would be tempting to expect that $j^{1}$ is still $H(\mu)$-hypermeasurable if the original $j$ was: however $g$ is not included in $M\left[j^{1}(g)\right]$ and $j^{1}$ is therefore just $\mu$-tall. There are some delicate issues involved if one wishes to preserve the $H(\mu)$-hypermeasurability of $\kappa$ in Theorem 2.1. A natural strategy is to prepare below $\kappa$ by a reverse Easton iteration. This approach is taken in [2] where it is also shown that if $\mu=\kappa^{++}$, then $Q$ is isomorphic to $\operatorname{Add}\left(\kappa^{+}, \kappa^{++}\right)$and thus the preparation can be implemented by iterating $\operatorname{Add}\left(\alpha^{+}, \alpha^{++}\right)$ at all inaccessible $\alpha \leq \kappa$. In [5], this representation is shown for $\mu=\kappa^{+n}$ for $2 \leq n<\omega$, i.e. $i\left(\operatorname{Add}\left(\kappa, \kappa^{+n}\right)\right)$ is isomorphic to $\operatorname{Add}\left(\kappa^{+}, \kappa^{+n}\right)$. It seems it is possible to continue up to the first cardinal above $\kappa$ with cofinality $\kappa$, but it is unclear whether it can be extended further.

Remark 2.3. The loss of the $H(\mu)$-hypermeasurability of $j^{1}$ may prevent the use of this method in more complicated situations (such as a subsequent definition of Radin forcing to achieve results of a more global character).

Let us work in the model $V[g]=V^{1}$ and let us use the notation $j^{1}, i^{1}, k^{1}, V^{1}, M^{1}, N^{1}$ to denote the resulting models and embeddings in Theorem 2.1. Using a fast-function forcing of Woodin, we can assume that there is $f: \kappa \rightarrow \kappa$ in $V$ such that $j(f)(\kappa)=\mu$. Let us denote $f(\alpha)$ by $\mu_{\alpha}$; let $C(f)$ denote the closed unbounded set of the closure points of $f:$ if $\alpha \in C(f)$, then for all $\beta<\alpha, f(\beta)<\alpha$.

Theorem 2.4. There is a forcing iteration $R_{\kappa}$ defined in $V^{1}$ such that

$$
V^{1}\left[R_{\kappa}\right][\operatorname{Add}(\kappa, \mu)] \vDash \kappa \text { is } \mu \text {-tall, }
$$


where $\operatorname{Add}(\kappa, \mu)$ is defined in $V\left[R_{\kappa}\right]$.

Proof. Define $R_{\kappa}$ to be the following Easton-supported iteration:

$$
\left.R_{\kappa}=\left\langle\left(R_{\alpha}, \dot{Q}_{\alpha}\right)\right| \alpha \in C(f), \alpha \text { inaccessible }\right\rangle,
$$

where $\dot{Q}_{\alpha}$ denotes the forcing $\operatorname{Add}\left(\alpha, \mu_{\alpha}\right)$.

The proof uses the usual surgery argument (see [3]) with Fact 2.5 which allows us to use the generic filter $g$ added in $V^{1}$ (for the $i^{1}$-image of $\operatorname{Add}(\kappa, \mu)^{1}$ ) in the model $V^{1}\left[R_{\kappa}\right]$ (for the proof, see Fact 2 in [2]). ${ }^{10}$

Fact 2.5. Let $S$ be a $\kappa$-cc forcing notion of cardinality $\kappa, \kappa^{<\kappa}=\kappa$. Then for any $\mu$, the term forcing $Q_{\mu}=\operatorname{Add}(\kappa, \mu)^{V[S]} / S$ is isomorphic to $\operatorname{Add}(\kappa, \mu)$.

Now we proceed with the proof of Theorem 2.4. Let $G_{\kappa} * H$ be $R_{\kappa} * \operatorname{Add}(\kappa, \mu)^{V^{1}\left[R_{\kappa}\right]_{-}}$ generic over $V^{1}$. Using the standard methods, $\operatorname{lift}^{11}$ in $V^{1}\left[G_{\kappa} * H\right]$ the embeddings $j^{1}, i^{1}, k^{1}$ to $R_{\kappa}$, obtaining commutative triangle $j^{1}: V^{1}\left[G_{\kappa}\right] \rightarrow M^{1}\left[j^{1}\left(G_{\kappa}\right)\right], i^{1}: V^{1}\left[G_{\kappa}\right] \rightarrow$ $N^{1}\left[i^{1}\left(G_{\kappa}\right)\right]$, and $k^{1}: N^{1}\left[i^{1}\left(G_{\kappa}\right)\right] \rightarrow M^{1}\left[j^{1}\left(G_{\kappa}\right)\right]$.

Using the elementarity of $i^{1}$, Fact 2.5 applied with $S=i^{1}\left(R_{\kappa}\right)$ and $i^{1}(\operatorname{Add}(\kappa, \mu))$ shows that $g$ - which is present in $V^{1}$ - yields a generic filter $g^{\prime}$ for the forcing $i^{1}(\operatorname{Add}(\kappa, \mu))$ of $N^{1}\left[i^{1}\left(G_{\kappa}\right)\right]$. The pointwise image of $g^{\prime}$ via $k^{1}$ generates a $j^{1}(\operatorname{Add}(\kappa, \mu))$-generic filter over $M^{1}\left[j^{1}\left(G_{\kappa}\right)\right]$, which is then modified by the standard surgery argument to allow for lifting $j^{1}$ to $V^{1}\left[G_{\kappa} * H\right]$ (for details see [2]); i.e. if we denote the lifting of $j^{1}$ by $j^{2}$, then

$$
j^{2}: V^{1}\left[G_{\kappa}\right][H] \rightarrow M^{1}\left[j^{1}\left(G_{\kappa} * H\right)\right]
$$

witnesses the measurability, and in fact $\mu$-tallness, of $\kappa$.

\section{Hypermeasurable cardinals}

It seems natural to extend Theorem 2.4 and have that the measurability of $\kappa$ ensured by $\operatorname{Add}(\kappa, \alpha)$ for any ordinal $\alpha, 0<\alpha \leq \mu$. We will show that this can be achieved with some additional assumptions on $\mu$. For concreteness, we will focus on the example where $\mu=\kappa^{+n}$ for some $1<n<\omega$.

First, in Theorem 3.1, we provide a standard construction which actually forces $\kappa$ to stop being measurable in $V^{*}$; the measurability of $\kappa$ is then resurrected by $\operatorname{Add}(\kappa, \alpha)$ for any $\kappa^{+} \leq \alpha \leq \kappa^{+n}$.

Theorem 3.1. (GCH) Let $1<n<\omega$ be fixed and assume $\kappa$ is $H\left(\kappa^{+n}\right)$-hypermeasurable. Then there is an iteration $P^{1}$ such that in $V\left[P^{1}\right]=V^{1}, \kappa$ is still $\kappa^{+n}$-hypermeasurable, and for some reverse Easton iteration $P_{\kappa}$ defined in $V^{1}, \kappa$ stops being measurable in $V^{*}=V^{1}\left[P_{\kappa}\right]$. In $V^{*}$, the measurability - in fact the hypermeasurability - of $\kappa$ is resurrected by Cohen forcing $\operatorname{Add}(\kappa, \alpha)$ for any $\kappa^{+} \leq \alpha \leq \kappa^{+n}$.

10 Recall that $Q_{\mu}$ - mentioned in Fact 2.5 - is the term forcing defined as follows: the elements of $Q_{\mu}$ are names $\tau$ such that $\tau$ is an $S$-name and it is forced by $1_{S}$ to be in $\operatorname{Add}(\kappa, \mu)$ of $V[S]$. The ordering is $\tau \leq \sigma \leftrightarrow 1_{S} \Vdash$ $\tau \leq \sigma$.

11 For simplicity, we use the notation $j^{1}, i^{1}, k^{1}$ to denote the partial liftings of the embeddings $j^{1}, i^{1}, k^{1}$. 
Proof. Let $j$ be an extender embedding witnessing the $H\left(\kappa^{+n}\right)$-hyper-measurability of $\kappa$, and let $i$ be a normal embedding generated by the normal measure $U$ derived from $j$. Recall Lemma 3.2 from [5] which implies that if $i: V \rightarrow N$ is an embedding generated by a normal measure on $\kappa$, then

$$
\operatorname{Add}\left(i(\kappa), i(\kappa)^{+n}\right)^{N} \cong \operatorname{Add}\left(\kappa^{+}, \kappa^{+n}\right) .
$$

Define $P^{1}$ is an Easton-supported iteration

$$
\left.\left\langle\left(P_{\alpha}^{1}, \dot{Q}_{\alpha}\right)\right| \alpha<\kappa, \alpha \text { is inaccessible }\right\rangle * \dot{Q}_{\kappa},
$$

where for an inaccessible $\beta \leq \kappa, \dot{Q}_{\beta}$ is $\operatorname{Add}\left(\beta^{+}, \beta^{+n}\right)$ of $V\left[P_{\beta}^{1}\right]$.

Let $G_{\kappa} * g$ be $P_{\kappa}^{1} * \dot{Q}_{\kappa}$-generic over $V$, and denote $V\left[G_{\kappa} * g\right]$ by $V^{1}$. Let $j^{1}$ and $i^{1}$ be the liftings of $j$ and $i$.

In $V^{1}$ define $P_{\kappa}$ as an Easton supported iteration:

$$
\left.P_{\kappa}=\left\langle\left(P_{\alpha}, \dot{Q}_{\alpha}\right)\right| \alpha<\kappa \text { is inaccessible }\right\rangle,
$$

where $\dot{Q}_{\alpha}$ denotes the forcing $\operatorname{Add}\left(\alpha, \alpha^{+n}\right)$ of $V^{1}\left[P_{\alpha}\right]$.

First note that $\kappa$ stops being measurable in $V^{*}=V^{1}\left[P_{\kappa}\right]$ by the application of the gap-forcing theorem of [6]: a hypothetical embedding $k$ with critical point $\kappa$ found in $V^{*}$ could be written as an embedding from $V^{1}\left[P_{\kappa}\right]$ to some $N\left[j\left(P_{\kappa}\right)\right]$, with $N \subseteq V^{1}$; in particular a generic filter for $j\left(P_{\kappa}\right)$ would need to add a non-trivial generic filter at stage $\kappa$ which cannot be found in $V^{1}\left[P_{\kappa}\right]$.

The rest of the Theorem follows from the following Claim:

Claim 3.2. Let $\alpha$ be an ordinal, $\kappa^{+} \leq \alpha \leq \kappa^{+n}$. Then $\kappa$ is still measurable in $V^{1}\left[P_{\kappa}\right]$ $[\operatorname{Add}(\kappa, \alpha)]$, where $\operatorname{Add}(\kappa, \alpha)$ is defined in $V^{1}\left[P_{\kappa}\right]$.

Proof. It suffices to show the Claim for $\alpha$ 's which are cardinals. So assume $\kappa^{+m}=|\alpha|$ for some $1 \leq m \leq n$. Choose in $V^{1}$ an embedding $j_{m}: V^{1} \rightarrow M_{m}$ which witnesses the $H\left(\kappa^{+m}\right)$-hypermeasurability of $\kappa$ with $\kappa^{+m}<j_{m}(\kappa)<\kappa^{+m+1}$ (this is possible since $2^{\kappa}=\kappa^{+}$

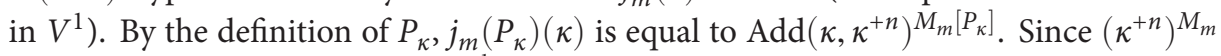

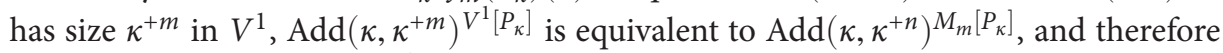

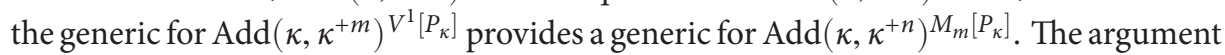
is then finished as in Theorem 2.4, using the fact that the generic $g$ for $i^{1}\left(\operatorname{Add}\left(\kappa, \kappa^{+n}\right)\right)$ is also generic for $i^{1}\left(\operatorname{Add}\left(\kappa, \kappa^{+m}\right)\right)$.

This concludes the proof of Theorem 3.1.

Note that the method in the proof of Theorem 3.1 does not work for the case of $\alpha$ smaller than $\kappa^{+}$: every elementary embedding $k: V^{1} \rightarrow M$ with critical point $\kappa$ sends

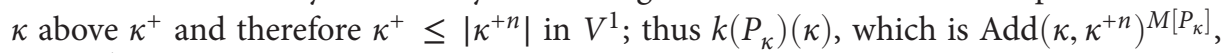
is in $V^{1}\left[P_{\kappa}\right]$ equivalent to the Cohen forcing at $\kappa$ of length at least $\kappa^{+}$. It follows that to lift the embedding, we need to force over $V^{1}\left[P_{\kappa}\right]$ with a Cohen forcing at $\kappa$ of length at least $\kappa^{+}$. If $\alpha<\kappa^{+}$, this condition is not satisfied. We remedy this by a more complicated construction in Theorem 3.3.

Theorem 3.3. With the assumptions and the notation as in Theorem 3.1, one can define $P_{\kappa}$ so that $\kappa$ is measurable in $V^{*}$, and its measurability - in fact hypermeasurability - is indestructible by $\operatorname{Add}(\kappa, \alpha)$ for any $0<\alpha \leq \kappa^{+n}$. 
Proof. Modify the definition of $P_{\kappa}$ in (3.3) so that at an inaccessible $\alpha<\kappa, \dot{Q}_{\alpha}$ is chosen generically ${ }^{12}$ amongst the following forcings: $\{1\}$ (the trivial forcing), and $\operatorname{Add}\left(\alpha, \alpha^{+k}\right)$, for $0 \leq k \leq m$.

Then one can argue that $\kappa$ is still measurable in $V^{*}$ : while lifting the embedding $j^{1}$, it suffices to work below a condition in $j^{1}\left(P_{\kappa}\right)$ which chooses the trivial forcing $\{1\}$ at stage $\kappa$.

To argue that for any $0<\alpha \leq \kappa^{+n}, \kappa$ is still measurable in $V^{*}[\operatorname{Add}(\kappa, \alpha)]$, work below a condition in $j^{1}\left(P_{\kappa}\right)$ which chooses the right forcing at stage $\kappa$.

\section{Open questions}

Q1. Is it possible to generalise Theorem 2.4 so that $\mu$ is still $H(\mu)$-hypermeasurable if the original embedding $j$ was $H(\mu)$-hypermeasurable? This would require some sort of preparation below $\kappa$ in the model $V^{1}$ (analogously to the methods in Theorem 3.1).

A related question is this:

Q2. Is it possible to characterise the forcings $i(\operatorname{Add}(\kappa, \mu))$, where $i: V \rightarrow N$ is a normal measure ultrapower as in Theorem 2.1? We know that this forcing does not collapse (it is $\kappa^{+}$-closed and $\kappa^{++}$-cc in $V$ ), but does it have a uniform representation? In particular, is it isomorphic to $\operatorname{Add}\left(\kappa^{+}, \mu\right)$ of $V$ ?

\section{Acknowledgments}

The work was mainly supported by FWF/GAČR grant I 1921-N25.

A support of travel grant Mobility 7AMB15AT035 is also acknowledged.

\section{References}

[1] Arthur W. Apter. Strong Cardinals can be Fully Laver Indestructible. Mathematical Logic Quarterly, 48: 499-507, 2002.

[2] James Cummings. A model in which GCH holds at successors but fails at limits. Transactions of the American Mahematical Society, 329(1): 1-39, 1992.

[3] James Cummings. Iterated forcing and elementary embeddings. In Matthew Foreman and Akihiro Kanamori, editors, Handbook of Set Theory, volume 2. Springer, 2010.

[4] James Cummings and Matthew Foreman. The tree property. Advances in Mathematics, 133(1): 1-32, 1998.

[5] Moti Gitik and Carmi Merimovich. Possible values for $2^{\aleph_{n}}$ and $2^{\aleph_{\omega}}$. Annals of Pure and Applied Logic, 90(1-3): 193-241, 1997.

[6] Joel David Hamkins. Gap forcing. Israel Journal of Mathematics, 125(1): 237-252, 2001.

[7] Richard Laver. Making the supercompactness of $\kappa$ indestructible under $\kappa$ directed closed forcing. Israel Journal of Mathematics, 29(4): 385-388, 1978.

12 The "lottery preparation" in the terminology of Hamkins. 